\title{
The prevalence, incidence, prognosis and risk factors for symptoms of depression and anxiety in a UK cohort during the COVID-19 pandemic
}

\author{
Ru Jia, Kieran Ayling, Trudie Chalder, Adam Massey, Norina Gasteiger, Elizabeth Broadbent, \\ Carol Coupland and Kavita Vedhara
}

\section{Background}

The COVID-19 pandemic has had profound consequences for population mental health. However, it is less clear for whom these effects are sustained.

\section{Aims}

To investigate the prevalence, incidence, prognosis and risk factors for symptoms of depression and anxiety in a UK cohort over three distinct periods in the pandemic in 2020

\section{Method}

An online survey was completed by a UK community cohort at three points ( $n=3097$ at baseline, $n=878$ completed all surveys): April (baseline), July to September (time point 2) and November to December (time point 3). Participants completed validated measures of depression and anxiety on each occasion, and we prospectively explored the role of sociodemographic and psychological factors (loneliness, positive mood and perceived risk of and worry about COVID-19) as risk factors.

\section{Results}

Depression (Patient Health Questionnaire-9 means: baseline, 7.69; time point 2, 5.53; time point $3,6.06$ ) and anxiety scores (Generalised Anxiety Disorder-7 means: baseline, 6.59; time point 2, 4.60; time point 3, 4.98) were considerably greater than pre-pandemic population norms at all time points. Women reported greater depression and anxiety symptoms than men.
Younger age, history of mental health disorder, more COVID-19related negative life events, greater loneliness and lower positive mood at baseline were all significant predictors of poorer mental health at time point 3.

\section{Conclusions}

The negative impact of the COVID-19 pandemic on mental health has persisted to some degree. Younger people and individuals with prior mental health disorders are at greatest risk. Easing of restrictions and resumption of social interaction could mitigate the risk factors of loneliness and positive mood.

\section{Keywords}

Mental health; depression; anxiety; risk factors; COVID-19.

\section{Copyright and usage}

(C) The Author(s), 2022. Published by Cambridge University Press on behalf of the Royal College of Psychiatrists. This is an Open Access article, distributed under the terms of the Creative Commons Attribution-NonCommercial-NoDerivatives licence (https://creativecommons.org/licenses/by-nc-nd/4.0/), which permits non-commercial re-use, distribution, and reproduction in any medium, provided the original work is unaltered and is properly cited. The written permission of Cambridge University Press must be obtained for commercial re-use or in order to create a derivative work.
The COVID-19 pandemic has resulted in unprecedented disruptions to people's daily lives, healthcare provision and the economy. There is a growing body of literature reporting evidence of a rapid and significant deterioration in the UK population's mental health, which occurred within weeks of the first national lockdown, a pattern repeated in many countries. ${ }^{1}$ However, the nature of the pandemic has changed over time, with levels of infection and mortality fluctuating, which, in turn, have precipitated changes in social restrictions. We describe here the prevalence, incidence and prognosis of mental health difficulties reported in a UK cohort established early in the pandemic, over three distinct periods in 2020.

The COVID-19 pandemic can be characterised as a chronic stressor, $^{2}$ in that it has now affected the lives of most people for more than a year. There is no clear end in sight, and it is both unpredictable and largely uncontrollable at the individual level. In the UK, the trajectory of the pandemic in 2020 has had several key phases. It commenced with the first national lockdown (23 March 2020), when people were instructed to stay at home and schools were closed. Aside from a small number of exceptions, for most of the UK, the lockdown was gradually eased from 11 May 2020, ${ }^{3}$ with people allowed to meet others from outside their household and the reopening of schools, hospitality and retail venues. This continued to early September 2020. However, from September 2020, the number of areas in which local restrictions were tightened began to increase, and the spiralling number of infections and deaths led inexorably to a second lockdown in November 2020, with many and fluctuating restrictions throughout December. One of the considerations of public health policy regarding the changes in social restrictions was, and continues to be, its impact on mental health. ${ }^{4}$ It is, therefore, important to examine whether mental health did indeed improve in response to eased restrictions and subsequently if a resumption of restrictions precipitated a deterioration.

Evidence from longitudinal studies with large UK cohorts suggests that levels of anxiety and depression, for example, improved during summer $2020,{ }^{5,6}$ but less is known about the impact of the autumn/winter lockdown. Beyond a simple description of how mental health has fluctuated in response to social restrictions, it is also of interest to examine whether the characteristics associated with mental health difficulties at the start of the pandemic remained consistent over time. For example, several studies demonstrated that young people and women were at greater risk of psychological distress early in the pandemic. ${ }^{5-7}$ In addition to these demographic predictors, we and others have reported that greater perceived risk of COVID-19, worry about contracting COVID-19, loneliness and reduced positive mood were also associated with greater depression and anxiety during lockdowns in different countries and regions. ${ }^{7-9}$ Finally, it is also relevant to examine the factors that predict how individuals' mental health changed in response to the pandemic. The seemingly sudden and rapid deterioration in mental health for large swathes of the population was perhaps not 
unexpected, considering that the pandemic was initially a novel and unprecedented experience for most people. However, stress and coping theory ${ }^{2}$ would lead us to expect that some people will have been able to adjust to the challenges of the pandemic by identifying and implementing effective coping strategies. ${ }^{10}$ Previous work from the severe acute respiratory syndrome (SARS) epidemic suggested that less SARS-related worry and greater social support were protective factors against subsequent mental health difficulties. ${ }^{11}$

\section{Hypotheses}

We report on these issues here by presenting analyses from a longitudinal community cohort from the UK. We hypothesised that (a) symptoms of anxiety and depression during the pandemic would exceed pre-pandemic population norms, and vary in response to different restrictions over the course of the study (first lockdown/ time point 1 (baseline), eased restrictions/time point 2 , increased restrictions/time point 3); (b) sociodemographic (e.g. age) and psychological factors (e.g. loneliness) at baseline would predict anxiety and depression symptoms at time point 3; and (3) sociodemographic (e.g. age) and psychological factors (e.g. loneliness) would be associated with the incidence and prognosis of symptoms of depression and anxiety over time.

\section{Method}

\section{Recruitment and eligibility}

The authors assert that all procedures contributing to this work comply with the ethical standards of the relevant national and institutional committees on human experimentation and with the Helsinki Declaration of 1975, as revised in 2008. The University of Nottingham Faculty of Medicine and Health Sciences (approval number 506-2003) and the National Health Service (NHS) Health Research Authority (approval number 20/HRA/1858) approved all study procedures. Recruitment processes were reported previously. ${ }^{8}$ In short, participants were recruited in the community through a social and mainstream media campaign between 3 and 30 April 2020. NHS organisations were also approached to promote the research through their routine communications. Potential participants were directed to the study website, through which they accessed the information sheet, consent form and online survey.

Eligible participants were aged 18 years and over, able to give informed consent, able to read English, residing in the UK at the time of completing the survey and able to provide a sample of hair at least $1 \mathrm{~cm}$ long. The latter was collected for the determination of the stress biomarker cortisol, which will be the subject of future manuscripts.

\section{Procedures}

Consenting participants completed an online survey implemented through JISC Online Survey (https://www.onlinesurveys.ac.uk/). Data were collected on three occasions, as follows (Fig. 1): baseline, between 3 and 30 April 2020 (national lockdown); time point 2, between 1 July and 21 September 2020 (eased restrictions); and time point 3, between 11 November and 31 December 2020 (increased restrictions including 4 weeks of lockdown).

Participants who completed the baseline survey were invited by email to complete the survey again at time points 2 and 3. Sociodemographic factors (age, gender, ethnicity, keyworker status, being in a recognised COVID-19 risk category, living alone or with others) were collected at baseline. The following psychological measures were collected at all time points: anxiety (Generalised Anxiety Disorder-7 (GAD-7); $\alpha=0.88)^{12}$ and depression (Patient Health Questionnaire-9 (PHQ-9); $\alpha=0.92) .{ }^{13}$ We also assessed positive mood (Scale of Positive and Negative Experience, positive items; $\alpha=0.94$ ), ${ }^{14}$ worry about contracting COVID-19, perceived loneliness and risk of COVID-19, details of which are reported elsewhere. ${ }^{8}$ In addition, at time point 2 we asked participants whether they had prior mental health disorders, and at time point 3, we asked whether participants had experienced any negative/positive life events related to COVID-19 (based on a brief checklist of events) at some point since the start of the pandemic. Negative and positive life events were totalled and scored separately (for item details see Supplementary Appendix 1 available at https://doi.org/10.1192/bjo.2022.34).

\section{Statistical analysis}

We first summarised the outcome variables (depression and anxiety symptoms scores) and participant characteristics with appropriate descriptive statistics, and examined histograms and scatterplots for normality. Comparisons with pre-pandemic normative values were made with independent samples $t$-tests. Examination of histograms indicated that both depression and anxiety scores deviated from a normal distribution; however, transformations or non-parametric tests were not suitable for these comparisons because only summary statistics (not individual-level data) were available for normative data. Although $t$-tests are robust to deviations from normality, especially when sample sizes are large, ${ }^{15}$ results of these specific tests should be interpreted with caution. Depression and anxiety symptoms were also categorised based on original cut-offs. ${ }^{12,13}$

Comparisons of mental health outcomes at three time points were made with repeated measures ANOVA. We conducted multivariable linear regression models to explore the independent relationships of sociodemographic factors (age, gender, ethnicity, keyworker status, prior mental health disorders, living alone, being in a recognised COVID-19 risk group, experience of pandemic-related positive/negative life events) and baseline psychological factors (perceived loneliness, perceived risk of COVID-19, positive mood, COVID-19-related worry), with depression and anxiety symptoms scores at time point 3 . The variable assessing COVID-19-related worry was treated as a categorical variable in all models, with 'occasional worry' treated as the reference value as this was the most common response. Assumptions of linear regression (normality and homoscedasticity of residuals, linearity with continuous variables) and presence of outliers were assessed graphically. Square-root transformations were used for depression and anxiety symptoms scores to satisfy assumptions. Multicollinearity was checked for in all models, using variance inflation factors, and found to have acceptable levels (variance inflation factor $<10$ ).

To examine predictors of incidence and prognosis of depression and anxiety symptoms, we dichotomised depression and anxiety outcomes according to established cut-offs for 'caseness', ${ }^{16}$ where levels of symptoms reached the thresholds for high-intensity psychological support (PHQ-9 score $\geq 10$, GAD-7 score $\geq 8$ ) in the NHS. Cochran's $Q$-tests were conducted to examine the differences in the proportions of depression and anxiety 'cases' over time. Individuals who were not classified as cases of depression or anxiety at baseline, but became cases at time point 2 or 3 , were classified as incident depression or anxiety cases. Individuals who were classified as cases for depression or anxiety at baseline, but subsequently became non-cases at time point 2 or 3 , were further classified as remission of depression or anxiety cases. We used logistic regression to estimate odds ratios with $95 \%$ confidence intervals for associations with incidence and improvement of depression and anxiety cases at time point 3 , using demographic and psychological factors at baseline, relative to no change in case status. Demographic and psychological factors that were significantly associated with symptoms of 


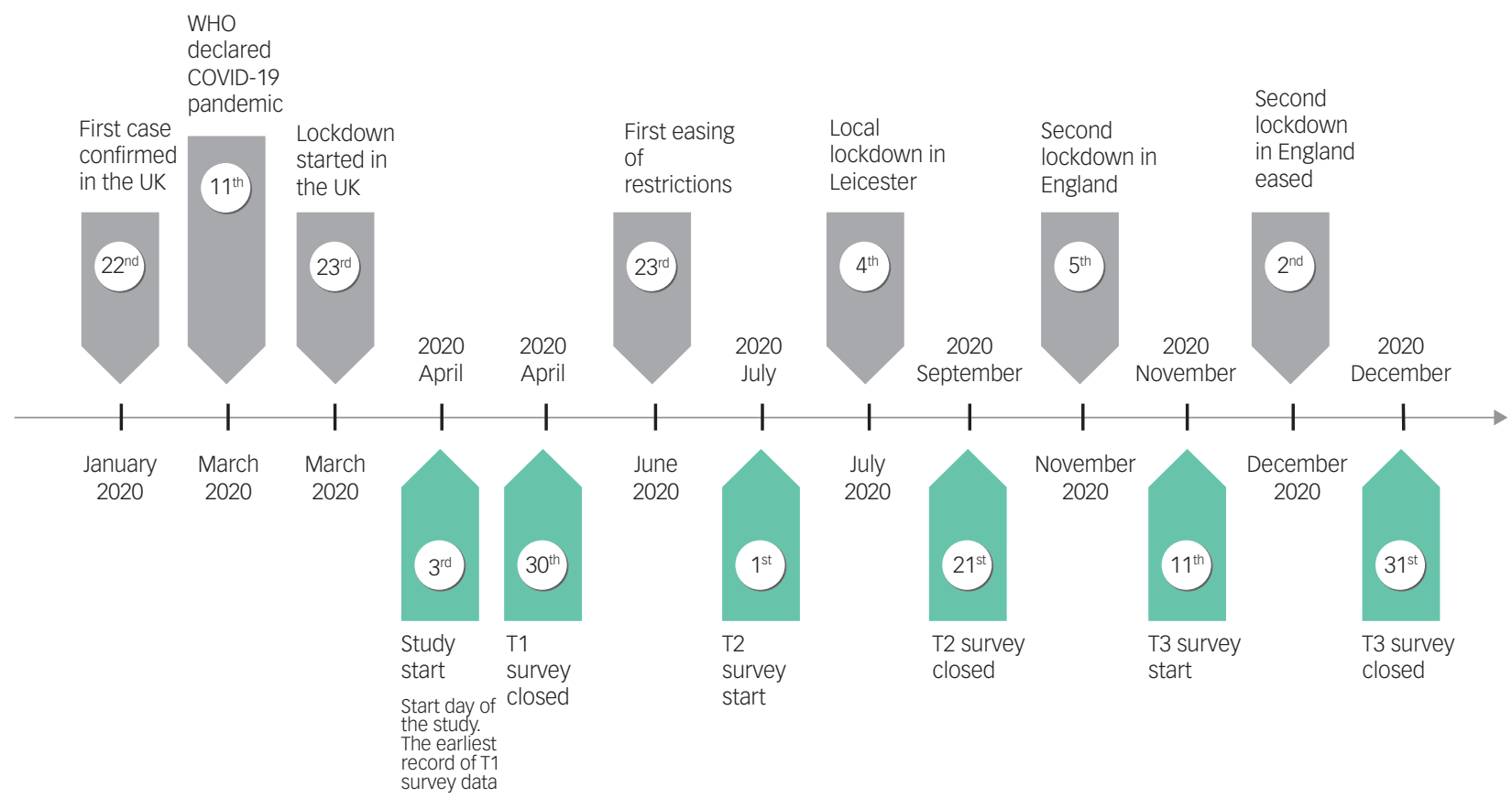

Fig. 1 Timeline of study data collection and COVID-19 restriction measures in the UK. T1, baseline; T2, time point 2; T3, time point 3; WHO, World Health Organization.

depression or anxiety in the previous multivariable linear regression models were all included in the logistic regression analysis.

\section{Sensitivity analyses}

We performed several sensitivity analyses to examine the robustness of the analyses outlined above. First, we weighted our sample to reflect the most recent UK age and gender distributions (Office for National Statistics, mid-year estimates 2019). ${ }^{17}$ These weights were then applied to mean score and prevalence estimates for symptoms of depression and anxiety (Supplementary Table 3.1), as well as added into multivariable regression models (see Supplementary Tables 3.2 and 3.3 for models). Second, we repeated multivariable models, but added the relevant depression or anxiety scores at baseline (to account for baseline associations, Supplementary Table 3.4); and third, we used backward stepwise selection to address concerns relating to potential multicollinearity (Supplementary Tables 3.5 and 3.6). Finally, to address issues relating to attrition, we conducted further analyses with multiple imputation and chained equations to impute values for variables with missing values (age, gender, ethnicity, prior mental health disorders, pandemic-related positive/negative life events, depression and anxiety symptoms scores at all time points). This approach is suitable for longitudinal data. ${ }^{18} \mathrm{We}$ generated 70 imputed data-sets. Multivariable regression models predicting depression and anxiety scores at time point 3 were built with multiple imputation data-sets, and estimates were combined using Rubin's rules (see Supplementary Table 3.8 for models). Perceived risk of COVID-19 was not significant in the main analyses and so was excluded from this sensitivity analysis.

Statistical analyses were performed with Stata for Windows (version 16).

\section{Results}

\section{Cohort characteristics}

At baseline, 3097 participants completed the survey. A total of 45\% $(n=1385)$ of this cohort returned the follow-up survey at time point 2 , and 35\% $(n=1087)$ returned the survey at time point 3 ; $28 \%(n=881)$ of respondents completed all three surveys. Three participants left the UK before time point 3 and so were removed from analyses. This resulted in a final cohort of 878 UK-dwelling participants who completed all three surveys (completers). Demographic and baseline mental health characteristics of the completers and non-completers of all three surveys are presented in Table 1.

Significant differences in demographic and baseline mental health characteristics were found between completers and non-completers. Specifically, individuals who were younger $(P<0.001)$, from ethnic minority backgrounds $(P<0.001)$, keyworkers $(P<0.001)$, not in a COVID-19 risk group $(P=0.002)$ and living with others $(P=0.02)$ were more likely to drop out from the study. Participants with poorer mental health characteristics at baseline were also more likely to drop out from the study. This included those with higher depression symptoms scores (mean: 8.37 v. 5.96, $P<0.001$ ), higher anxiety symptoms scores (mean: 7.16 v. $5.15, P<0.001$ ), greater loneliness scores (mean: $4.06 v .3 .33, P<0.001$ ), lower positive mood scores (mean: $18.56 v .20 .08, P<0.001$ ) and those who reported greater worry about getting COVID-19 $(P=0.002)$.

\section{Depression and anxiety symptoms over time}

Mean scores of depression and anxiety symptoms in the whole cohort at each time point are presented in Fig. 2. The overall mean values for depression and anxiety symptoms were significantly higher than previously reported population norms, ${ }^{19,20}$ at all three time points (all $P<0.001$ ). Female participants reported significantly higher levels of both depression and anxiety symptoms than male participants across time (depression symptoms: baseline, $P<0.001$; time point 2, $P<0.001$; time point $3, P=0.001$; anxiety symptoms: baseline, $P<0.001$; time point $2, P<0.001$; time point $3, P=0.002$ ). The mean depression and anxiety symptoms scores for both genders were also significantly higher than their respective population norms (all $P<0.001$ ). Mean depression and anxiety symptoms scores weighted to reflect the most recent UK age and gender distributions were lower than unweighted results, but 
Table 1 Baseline characteristics in completers and non-completers

\begin{tabular}{|c|c|c|}
\hline & $\begin{array}{c}\text { Completers, } \\
n(\%)\end{array}$ & $\begin{array}{c}\text { Non-completers } \\
n(\%)\end{array}$ \\
\hline N & $878(28.4 \%)$ & $2216(71.6 \%)$ \\
\hline \multicolumn{3}{|l|}{ Gender } \\
\hline Male & $123(14.0 \%)$ & $353(15.9 \%)$ \\
\hline Female & 754 (85.4\%) & 1861 (84.0\%) \\
\hline Prefer not to say & $1(0.1 \%)$ & $2(0.1 \%)$ \\
\hline Age, mean (s.d.) $)^{\mathrm{a}}$ & $49.7(15.0)$ & $42.6(14.5)$ \\
\hline \multicolumn{3}{|l|}{ Age groups, years } \\
\hline $18-24$ & 49 (5.6\%) & $313(14.1 \%)$ \\
\hline $25-34$ & $117(13.3 \%)$ & $410(18.5 \%)$ \\
\hline $35-44$ & $147(16.7 \%)$ & $490(22.1 \%)$ \\
\hline $45-54$ & $193(22.0 \%)$ & $497(22.5 \%)$ \\
\hline $55-64$ & $218(24.8 \%)$ & 352 (15.9\%) \\
\hline $65-74$ & $129(14.7 \%)$ & $128(5.8 \%)$ \\
\hline$\geq 75$ & $25(2.9 \%)$ & $24(1.1 \%)$ \\
\hline \multicolumn{3}{|l|}{ Ethnicity $^{\mathrm{a}}$} \\
\hline White British, Irish, other & $826(94.1 \%)$ & 1967 (88.9\%) \\
\hline BAME background & $51(5.8 \%)$ & 245 (11.1\%) \\
\hline \multicolumn{3}{|l|}{ Keyworker status ${ }^{a}$} \\
\hline Keyworker & 354 (40.3\%) & 1204 (54.3\%) \\
\hline Not a keyworker & $524(59.7 \%)$ & $1012(45.7 \%)$ \\
\hline \multicolumn{3}{|l|}{ COVID-19 risk groups ${ }^{a}$} \\
\hline $\begin{array}{l}\text { Most at risk (e.g. suffering } \\
\text { from advanced cancer, } \\
\text { severe asthma/COPD, } \\
\text { etc.) }\end{array}$ & 25 (2.9\%) & $96(4.3 \%)$ \\
\hline $\begin{array}{l}\text { At increased risk (e.g. } \\
\text { being pregnant, aged } \\
>70 \text { years) }\end{array}$ & $180(20.5 \%)$ & $348(15.7 \%)$ \\
\hline Not at risk & $673(76.7 \%)$ & $1772(80.0 \%)$ \\
\hline \multicolumn{3}{|l|}{ Living alone or with others ${ }^{a}$} \\
\hline Living alone & 134 (15.3\%) & 271 (12.2\%) \\
\hline Living with others & 744 (84.7\%) & $1945(87.8 \%)$ \\
\hline $\begin{array}{l}\text { Depression symptoms, } \\
\text { mean (s.d.) }\end{array}$ & $5.96(5.2)$ & $8.37(6.2)$ \\
\hline $\begin{array}{l}\text { Anxiety symptoms, mean } \\
(\text { s.d. })^{\text {a }}\end{array}$ & $5.15(5.0)$ & $7.16(5.7)$ \\
\hline Loneliness, mean (s.d.) ${ }^{\mathrm{a}}$ & $3.33(2.5)$ & $4.1(2.8)$ \\
\hline Positive mood, mean (s.d. $)^{a}$ & $20.08(4.9)$ & $18.6(5.1)$ \\
\hline $\begin{array}{l}\text { Perceived risk of COVID-19, } \\
\text { mean (s.d.) }\end{array}$ & $3.93(1.87)$ & $4.9(2.3)$ \\
\hline \multicolumn{3}{|l|}{ COVID-19-related worry, $n(\%)^{\mathrm{a}}$} \\
\hline No worry & $191(21.8 \%)$ & 359 (16.2\%) \\
\hline Occasional worry & 626 (71.3\%) & 1443 (65.1\%) \\
\hline Much worry & $50(5.7 \%)$ & $311(14.0 \%)$ \\
\hline Most worry & $11(1.3 \%)$ & $103(4.7 \%)$ \\
\hline
\end{tabular}

higher than population norms (Supplementary Table 3.1). Sensitivity analysis using multiple imputation estimated higher mean values for depression symptoms (time point 2: 6.44, s.d. 0.13; time point 3: 6.83; s.d. 0.20 ) and anxiety (time point 2: 5.42 , s.d. 0.13 ; time point $3: 5.80$, s.d. 0.16 ) than the cross-sectional mean values reported in Fig. 2.

When comparing symptoms of depression and anxiety among completers, significant improvements were seen at time point 2 (depression, $P<0.001$; anxiety, $P<0.001$ ). Specifically, mean depression and anxiety scores (square-root transformed) were highest at baseline compared with time points 2 (both $P<0.001$ ) and 3 (depression, $P=0.002$; anxiety, $P<0.001$ ), whereas levels at time point 2 were not significantly different from time point 3 (depression, $P=0.10$; anxiety, $P=0.054$ ). Similarly, the cases of depression and anxiety according to the original cut-offs ${ }^{12,13}$ showed that fewer completers reported symptoms of depression $(49 \%)$ and anxiety $(37 \%)$ at time point 2 compared with baseline and time point 3 (Table 2). A similar pattern was found for caseness of depression and anxiety (Table 2). The prevalence of depression and anxiety were $21 \%$ and $24 \%$, respectively, at baseline. At time point 2, the prevalence of depression was $17 \%$, which was significantly lower than at baseline $(21 \%, P=0.005)$, but not at time point $3(19 \%, P=0.086)$. The prevalence of anxiety at time point 2 was $19 \%$, which was significantly lower than both baseline $(24 \%, P=0.001)$ and time point $3(22 \%, P=0.048)$. The prevalence of cases of depression and anxiety weighted to reflect the most recent UK age and gender distributions were similar to unweighted results (Supplementary Table 3.1).

\section{Examining risk factors for symptoms of depression and anxiety}

Multivariable linear regression models were constructed to identify sociodemographic and psychological predictors of depression and anxiety symptoms scores (square-root transformed) at time point 3 (Table 3). Results showed that being younger (depression scores: mean difference $-0.14,95 \%$ CI -0.20 to -0.09 ; anxiety scores: mean difference $-0.15,95 \% \mathrm{CI}-0.21$ to -0.09 ; both per 10-year increase), having a history of a mental health disorder (depression scores: mean difference $0.56,95 \%$ CI $0.41-0.72$; anxiety scores: mean difference $0.51,95 \%$ CI $0.35-0.68$ ) and reporting more pandemic-related negative life events since the start of the pandemic (depression scores: mean difference $0.24,95 \% \mathrm{CI}$ $0.16-0.32$; anxiety scores: mean difference 0.19 , 95\% CI $0.11-$ 0.28 ) were independently and significantly associated with greater depression and anxiety scores at time point 3 . In addition, living alone (mean difference $-0.44,95 \%$ CI -0.67 to -0.22 ) was
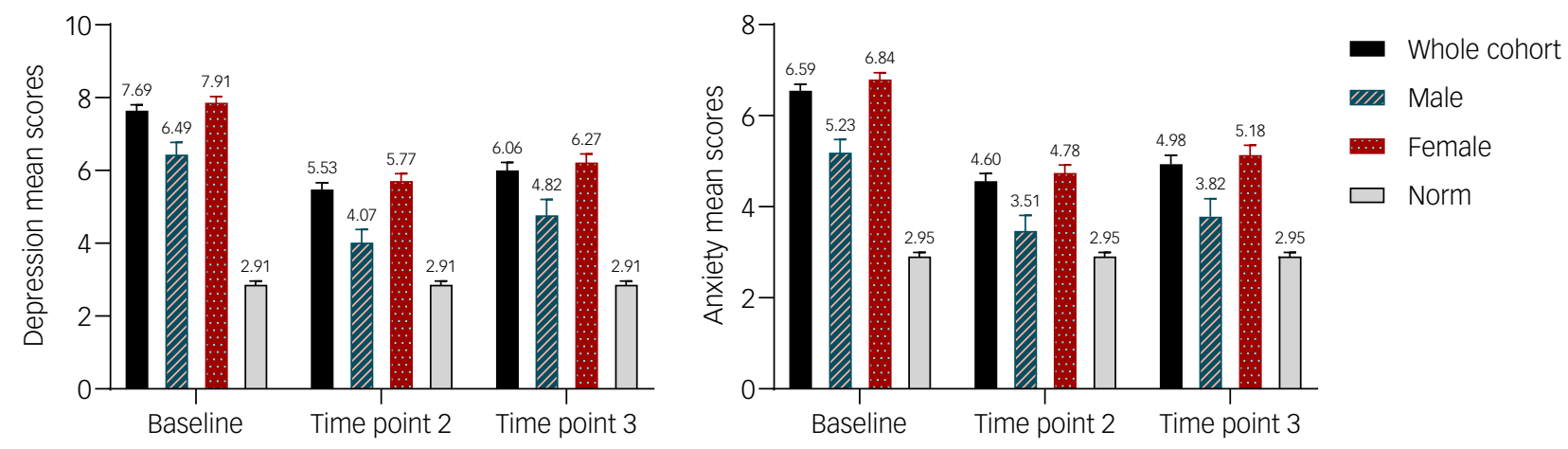


\begin{tabular}{|c|c|c|c|c|}
\hline & Categories & $\begin{array}{c}\text { Baseline } \\
n(\%)\end{array}$ & $\begin{array}{c}\text { Time point } 2 \\
n(\%)\end{array}$ & $\begin{array}{c}\text { Time point } 3 \\
n(\%)\end{array}$ \\
\hline \multicolumn{5}{|l|}{ Depression (PHQ-9) } \\
\hline \multirow{5}{*}{ Categories } & None/minimal Depression (0-4) & $428(48.8)$ & $470(53.5)$ & $440(50.1)$ \\
\hline & Mild depression (5-9) & $267(30.4)$ & $260(29.6)$ & $270(30.8)$ \\
\hline & Moderate depression (10-14) & $119(13.6)$ & $97(11.1)$ & $98(11.2)$ \\
\hline & Moderately severe depression (15-19) & $43(4.9)$ & $34(3.9)$ & $47(5.4)$ \\
\hline & Severe depression (20-27) & $21(2.4)$ & $17(1.9)$ & $23(2.6)$ \\
\hline \multirow[t]{2}{*}{ Cases $^{a}$} & Non-cases (0-9) & $695(79.2)$ & $730(83.1)$ & $710(80.9)$ \\
\hline & Cases (10-27) & $183(20.8)$ & $148(16.9)$ & $168(19.1)$ \\
\hline \multirow[t]{2}{*}{ Case incidence and improvement ${ }^{\mathrm{b}}$} & Incidence & Not applicable & $59(6.7)$ & $48(5.5)$ \\
\hline & Improvement & Not applicable & $94(10.7)$ & $21(2.4)$ \\
\hline \multicolumn{5}{|l|}{ Anxiety (GAD-7) } \\
\hline \multirow[t]{4}{*}{ Categories } & None/minimal Anxiety (0-4) & $493(56.2)$ & 552 (62.9) & $510(58.1)$ \\
\hline & Mild anxiety (5-9) & 239 (27.2) & $216(24.6)$ & $223(25.4)$ \\
\hline & Moderate anxiety (10-14) & $78(8.9)$ & $68(7.7)$ & $91(10.4)$ \\
\hline & Severe anxiety (15-21) & $68(7.7)$ & $42(4.8)$ & $54(6.2)$ \\
\hline \multirow[t]{2}{*}{ Cases $^{a}$} & Non-cases (0-7) & $671(76.4)$ & $710(80.9)$ & $688(78.4)$ \\
\hline & Cases (8-21) & $207(23.6)$ & $168(19.1)$ & $190(21.6)$ \\
\hline \multirow[t]{2}{*}{ Case incidence and improvement ${ }^{b}$} & Incidence & Not applicable & $53(6.0)$ & $45(5.1)$ \\
\hline & Improvement & Not applicable & $92(10.5)$ & $24(2.7)$ \\
\hline
\end{tabular}

Table 3 Multivariable linear regression models showing associations between demographic and psychological explanatory variables at baseline and depression and anxiety scores at time point 3

\begin{tabular}{|c|c|c|}
\hline & $\begin{array}{l}\text { Depression total score at time point } 3^{\text {a }} \\
\text { Mean difference }(95 \% \mathrm{Cl}), P \text {-value }\end{array}$ & $\begin{array}{l}\text { Anxiety total score at time point } 3^{\text {a }} \\
\text { Mean difference }(95 \% \mathrm{Cl}), P \text {-value }\end{array}$ \\
\hline Age, per 10-year increase & $-0.14(-0.20$ to 0.09$),<0.001$ & $-0.15(-0.21$ to -0.09$),<0.001$ \\
\hline Female, yes/no & $0.04(-0.16$ to 0.25$), 0.67$ & 0.11 (-0.11 to 0.33$), 0.33$ \\
\hline Black and minority ethnic background, yes/no & $-0.17(-0.49$ to 0.14$), 0.28$ & $-0.10(-0.44$ to 0.23$), 0.55$ \\
\hline Keyworker, yes/no & $0.09(-0.07$ to 0.25$), 0.28$ & $0.10(-0.06$ to 0.27$), 0.22$ \\
\hline Prior mental health disorder, yes/no & $0.56(0.41-0.72),<0.001$ & $0.51(0.35-0.68),<0.001$ \\
\hline \multicolumn{3}{|l|}{ Risk group ${ }^{b}$} \\
\hline Most at risk & $0.25(-0.18$ to 0.68$), 0.26$ & $0.11(-0.35$ to 0.56$), 0.64$ \\
\hline Increased risk & $0.14(-0.04$ to 0.33$), 0.13$ & $0.07(-0.13$ to 0.27$), 0.47$ \\
\hline Living alone, yes/no & $-0.16(-0.37$ to 0.06$), 0.15$ & $-0.44(-0.67$ to -0.22$),<0.001$ \\
\hline Pandemic-related positive life event, per unit & $-0.01(-0.12$ to 0.10$), 0.88$ & $-0.00(-0.12$ to 0.12$), 0.95$ \\
\hline Pandemic-related negative life event, per unit & $0.24(0.16-0.32),<0.001$ & $0.19(0.11-0.28),<0.001$ \\
\hline Baseline perceived loneliness, per unit & $0.08(0.04-0.11),<0.001$ & $0.05(0.02-0.09), 0.005$ \\
\hline Baseline positive mood, per unit & $-0.08(-0.10$ to 0.06$),<0.001$ & $-0.07(-0.09$ to -0.06$),<0.001$ \\
\hline Baseline perceived risk of COVID-19, per unit & $0.01(-0.02$ to 0.05$), 0.47$ & 0.01 (-0.03 to 0.05$), 0.59$ \\
\hline \multicolumn{3}{|l|}{ Baseline COVID-19-related worryc ${ }^{c}$} \\
\hline No worry & $-0.05(-0.25$ to 0.15$), 0.63$ & $-0.11(-0.33$ to 0.10$), 0.30$ \\
\hline Much of the time & $0.10(-0.14$ to 0.33$), 0.42$ & $0.25(-0.00$ to 0.50$), 0.05$ \\
\hline Most of the time & $-0.10(-0.63$ to 0.43$), 0.70$ & $0.16(-0.40$ to 0.72$), 0.58$ \\
\hline Adjusted $R^{2}$ & 0.39 & 0.33 \\
\hline N & 717 & 717 \\
\hline \multicolumn{3}{|c|}{$\begin{array}{l}\text { Bold text indicates statistical significance. } \\
\text { a. A square-root transformation was applied to the dependent variable. } \\
\text { b. Comparison reference group 'I am in neither risk category'.' } \\
\text { c. Comparison reference group 'I occasionally worry about getting coviD-19'. }\end{array}$} \\
\hline
\end{tabular}

significantly associated with lower anxiety scores. The sociodemographic predictors accounted for $23-24 \%$ of the variance (Supplementary Table 2.1). Greater perceived loneliness (depression scores: mean difference $0.08,95 \%$ CI $0.04-0.11$; anxiety scores: mean difference $0.05,95 \%$ CI $0.02-0.09$ ) and lower positive mood (depression scores: mean difference $-0.08,95 \% \mathrm{CI}-0.10$ to -0.06 ; anxiety scores: mean difference $-0.07,95 \%$ CI -0.09 to $-0.06)$ at baseline were independently and significantly associated with both greater depression and anxiety scores at time point 3 . Sensitivity analyses controlling for baseline depression and anxiety scores showed similar results, except that baseline loneliness was no longer a significant predictor of depression or anxiety scores at time point 3 , and baseline positive mood was no longer a significant predictor of anxiety scores at time point 3 (Supplementary Table 3.4).

The same regression models were weighted to reflect the UK age and gender distribution, and results remained largely the same (Supplementary Table 3.2). Sensitivity analyses with backward stepwise selection also showed similar results with age, previous mental health disorders, pandemic-related negative life events, baseline loneliness and baseline positive mood remaining as significant predictors in both models (Supplementary Tables 3.5 and 3.6). 


\begin{tabular}{|c|c|c|c|c|}
\hline & $\begin{array}{l}\text { Incident depression } \\
\qquad \text { cases }^{\text {b }} \\
\text { Odds ratio }(95 \% \mathrm{Cl}) \text {, } \\
\quad P \text {-value }\end{array}$ & $\begin{array}{c}\text { Incident anxiety cases }{ }^{\text {b }} \\
\text { Odds ratio }(95 \% \mathrm{Cl}) \text {, } \\
P \text {-value }\end{array}$ & $\begin{array}{l}\text { Improved depression } \\
\text { cases }^{\mathrm{c}} \\
\text { Odds ratio }(95 \% \mathrm{Cl}) \text {, } \\
\quad P \text {-value }\end{array}$ & $\begin{array}{c}\text { Improved anxiety cases } \\
\text { Odds ratio }(95 \% \mathrm{Cl}) \text {, } \\
P \text {-value }\end{array}$ \\
\hline Age, per 10-year increase & $1.06(0.87-1.29), 0.57$ & $0.78(0.64-0.94), 0.009$ & $1.12(0.85-1.48), 0.42$ & $1.01(0.79-1.28), 0.96$ \\
\hline Prior mental health disorder, yes/no & $3.17(1.83-5.47),<0.001$ & $\begin{array}{c}3.93(2.30-6.72) \\
<0.001\end{array}$ & $0.78(0.39-1.58), 0.49$ & $0.66(0.35-1.27), 0.22$ \\
\hline Live alone, yes/no & $0.54(0.25-1.18), 0.13$ & $0.38(0.15-0.94), 0.04$ & $1.44(0.57-3.68), 0.44$ & 3.48 (1.31-9.23), 0.012 \\
\hline $\begin{array}{l}\text { Pandemic-related negative life events, } \\
\text { per unit }\end{array}$ & 1.35 (1.04-1.76), 0.02 & 1.50 (1.17-1.92), 0.001 & $0.66(0.47-0.91), 0.011$ & $0.76(0.54-1.07), 0.12$ \\
\hline Baseline perceived loneliness, per unit & $1.31(1.16-1.49),<0.001$ & 1.10 (0.97-1.25), 0.12 & $0.92(0.81-1.05), 0.22$ & $0.84(0.74-0.96), 0.008$ \\
\hline Baseline positive mood, per unit & $0.90(0.84-0.96), 0.003$ & $0.92(0.86-0.98), 0.008$ & $1.05(0.96-1.15), 0.26$ & 1.03 (0.94-1.12), 0.54 \\
\hline Pseudo $R^{2}$ & 0.17 & 0.15 & 0.06 & 0.07 \\
\hline N & 652 & 638 & 152 & 177 \\
\hline \multicolumn{5}{|c|}{$\begin{array}{l}\text { Bold text indicates statistical significance. } \\
\text { a. A case is defined as a Patient Health Questionnaire-9 score } \geq 10 \text { for depression or Generalised Anxiety Disorder- } 7 \text { score } \geq 8 \text { for anxiety, at which level someone would qualify for high- } \\
\text { intensity psychological support. } \\
\text { b. Incidence refers to individuals who were non-cases at baseline and subsequently became cases at time point } 2 \text { or } 3 \text {. The comparison groups stayed non-cases of depression at all time and } \\
\text { non-cases of anxiety at all } 3 \text { time points, respectively. } \\
\text { c. Improvement refers to individuals who were cases at baseline and subsequently became non-cases at time point } 2 \text { or } 3 \text {. The comparison groups stayed cases of depression at all time and } \\
\text { cases of anxiety at all time, respectively. }\end{array}$} \\
\hline
\end{tabular}

Sensitivity analyses with multiple imputation data $(n=70$ imputations) showed that all of these predictors (age, prior mental health disorders, pandemic-related negative life events, loneliness, positive mood) remained significant in models predicting time point 3 depression or anxiety scores (Supplementary Table 3.8). However, in the multiple imputation sensitivity analyses, being female (mean difference $0.17,95 \%$ CI $0.01-0.33$ ) and having more COVID-19related worry (most of the time; mean difference $0.40,95 \%$ CI 0.04-0.75) also significantly predicted greater anxiety symptoms at time point 3 (mean difference $0.17,95 \%$ CI $0.01-0.33$ ), and experiencing fewer pandemic-related positive life events since the start of the pandemic significantly predicted greater symptoms of depression (mean difference $-0.20,95 \%$ CI -0.26 to -0.13 ) and anxiety (mean difference $-0.16,95 \% \mathrm{CI}-0.23$ to -0.09 ) at time point 3 .

\section{Cases of depression and anxiety: predictors of change over time}

We distinguished between those who became incident cases of depression and anxiety (i.e. did not meet the criterion for highintensity support at baseline, but did so at either time point 2 or 3 ) and those who improved (i.e. met criterion for high-intensity support at baseline, but not at time point 2 or 3 ). At follow-up, $n=70$ (3\%) people who were non-cases at baseline became incident depression cases, and $n=71$ (3\%) became incident anxiety cases. In contrast, $n=588(67 \%)$ and $n=573(65 \%)$ remained non-cases of depression and anxiety respectively. Risk factors for becoming an incident case were having prior mental health disorder (depression: odds ratio $3.17,95 \%$ CI 1.83-5.47; anxiety: odds ratio 3.93, 95\% CI 2.30-6.72), experiencing more pandemic-related negative life events since the start of the pandemic (depression: odds ratio $1.35,95 \% \mathrm{CI}$ 1.04-1.76; anxiety: odds ratio $1.50,95 \%$ CI $1.17-1.92)$ and lower baseline positive mood (depression: odds ratio $0.90,95 \% \mathrm{CI}$ 0.84-0.96; anxiety: odds ratio $0.92,95 \%$ CI $0.86-0.98$ ).

Greater baseline loneliness was significantly associated with higher risk of incident depression (odds ratio 1.31, 95\% CI 1.16-1.49), whereas being older (odds ratio $0.78,95 \%$ CI $0.64-0.94$ per 10 -year increase) and living alone (odds ratio 0.38 , 95\% CI 0.15-0.94) were significantly associated with a reduced risk of incident anxiety (Table 4 ).

There were 85 people (9\%) who were depression cases at baseline and $88(8 \%)$ people who were anxiety cases who improved during follow-up (Table 2). Compared with those who remained a case of depression $(n=68)$ or anxiety $(n=91)$ at all time points, experiencing fewer pandemic-related negative life events (odds ratio $0.66,95 \% \mathrm{CI}$ $0.47-0.91$ ) was a significant predictor of improved depression cases. Living alone (odds ratio 3.48, 95\% CI 1.31-9.23) but feeling less loneliness at baseline (odds ratio $0.84,95 \%$ CI $0.74-0.96$ ) were significant predictors of improved anxiety cases (Table 4 ). Sensitivity analysis for incidence and improvement of depression and anxiety cases weighted to reflect the UK age and gender distributions were also conducted, and results remained largely the same (Supplementary Tables 3.1 and 3.3).

\section{Discussion}

We report findings from a prospective cohort study established early in the COVID-19 pandemic in the UK to assess the impact of the pandemic on mental health over the course of 2020 .

\section{Mental health during the CoVID-19 pandemic}

Our findings supported the hypothesis that mean scores for depression and anxiety in our cohort would significantly exceed pre-pandemic population norms at all surveyed points. Between baseline and time point $3,17-21 \%$ of the cohort reported symptoms of depression and 19-24\% reported symptoms of anxiety that reached the threshold for high-intensity psychological support. Female participants also reported higher scores on our measures of depression and anxiety than male participants throughout our observation period. We also observed changes in levels of depression and anxiety symptoms at different surveyed points, reflecting different social restrictions in the UK at three key stages (first lockdown/baseline, eased restrictions/time point 2 , increased restrictions/time point 3 ), in support of our hypotheses.

Although significant improvements in symptoms of depression and anxiety were evident after the easing of restrictions (time point 2) compared with baseline, we should view these results with caution given the significant loss to follow-up in this study, which was characterised by participants who reported greater symptoms of depression and anxiety, loneliness and worry about COVID-19, as well as lower positive mood, being more likely to drop out from the study. To address this 'survivorship bias', ${ }^{21}$ we estimated means and prevalence of depression and anxiety symptoms at 
both time points 2 and 3, using multiple imputation. Estimated mean values for depression symptoms were observed to be higher than the cross-sectional mean values reported in main analysis. Estimated prevalence for depression symptoms (24-27\%) and anxiety symptoms (22-26\%) during follow-up was also higher than reported in the main analysis with the original cohort $(n=878)$, which are similar to figures reported in other work. ${ }^{6}$ These findings together demonstrate the profound disruptions to mental health associated with the COVID-19 pandemic and subsequent social restrictions, which may be prolonged and further influence the physical health of the population. ${ }^{6}$

\section{Predictors of mental health}

We also hypothesised that sociodemographic and psychological factors would predict these mental health outcomes. When looking at which sociodemographic factors were predictive of symptoms of depression and anxiety later in the pandemic, we found that age continued to be the most important demographic predictor. ${ }^{8}$ Having a prior mental health disorder was also a significant predictor, again shown by others. ${ }^{6,22}$ Among individuals who were non-cases at baseline, those with prior mental health disorders were three to four times more likely to reach the threshold for high-intensity psychological support during follow-up compared with those without such a history. The considerable disruption to mental health services because of the pandemic might have contributed to the worsened symptoms in this group. ${ }^{23,24}$ Urgent efforts are needed to investigate how to deliver adequate support to those who are in need, and how to prevent further deterioration of those with mental health difficulties. ${ }^{25,26}$

The experience of pandemic-related negative life events was another significant risk factor for greater symptoms of depression and anxiety symptoms later in the pandemic. In contrast, experiencing fewer pandemic-related negative life events since the start of the pandemic was the only significant predictor of improved depression symptoms. Other studies have also reported the presence of pandemic-related negative life events, such as illness and financial difficulties owing to COVID-19, as being significantly associated with increased symptoms of depression and anxiety. ${ }^{27,28}$ Interestingly, unlike pandemic-related negative life events, experiencing pandemic-related positive life events was not associated with depression and anxiety scores at time point 3 . This could be because of the emotional benefits of positive life events potentially being attenuated during a chronic stressor such as a pandemic. Indeed, there was some evidence of this in our cohort, with individuals reporting fewer positive events overall ( $57 \%$ of the cohort reported pandemic-related negative life events and only $26 \%$ reported positive life events). Conversely, it is plausible that the benefits of positive life events are felt not on negative moods, but other outcomes, such as positive mood. Both areas would be worthy of further enquiry. The prominence of negative life events in our findings, however, suggests that as we emerge from the pandemic, strategies to improve mental well-being should involve psychological support for managing mental health in combination with changes in infrastructure to reverse the harms caused by a stalling economy, overwhelmed health and social care services, gaps in educational attainment, etc. ${ }^{29,30}$

When exploring modifiable psychological risk factors of depression and anxiety symptoms, we found that greater loneliness and lower positive mood at baseline significantly predicted greater depression and anxiety symptoms in November/December 2020, as seen cross-sectionally in April 2020, ${ }^{8}$ after controlling for sociodemographic factors. The findings were sustained in backward stepwise sensitivity analyses designed to address potential multicollinearity. Loneliness and low positive mood have also been identified as risk factors for poorer mental health in other studies. ${ }^{9,31}$
We also found an $8-10 \%$ decrease in the odds of depression or anxiety cases incidence during follow-up, per unit increase in baseline positive mood. This was despite the absence of a relationship between positive mood and improved depression or anxiety symptoms. Together, these findings highlight the potential effects of improving social support (and in so doing, reducing loneliness) and positive mood on reducing the experience of symptoms of depression and anxiety. Positive psychological interventions, featuring elements such as mindfulness, gratitude and 'best possible self, could be among the armoury of approaches we take to address both loneliness and positive mood. ${ }^{32,33}$ Other approaches are also available, such as enhancing social skills and social support, relaxation and creative activities, all of which have been shown to improve these outcomes. ${ }^{32,33}$

\section{Limitations}

The current findings are derived from an opportunistic self-selected cohort. As such, it is possible that individuals who were interested in mental health and/or perceived themselves to be most affected by the pandemic may have been more likely to participate. It should, however, be noted that our sensitivity analysis weighted to the UK population age/gender distribution estimated similar results to those reported in the main analysis. Second, we used self-reported psychological scales to measure depression and anxiety symptoms rather than clinical diagnosis data, thus we can only comment on the symptoms of mental health difficulties rather than the presence of clinically verified psychiatric disorders.

Third, although the drop-out rate $(72 \%)$ in our study was comparable to other cohorts established early in the pandemic, ${ }^{21,34}$ we observed important differences in demographic and psychological indices between people who dropped out and those who remained in the study. To address this, we conducted sensitivity analyses using multiple imputation for all models. The results remained largely the same, with age, prior mental health disorders, pandemic-related negative life events, loneliness and positive mood continuing to predict symptoms of depression and anxiety symptoms at time point 3 . This suggested that these models were unlikely to be affected by attrition. However, the large attrition is nonetheless likely to have led to an underestimation of depression and anxiety symptoms in our cohort. This was supported by the higher estimated means and prevalence of depression and anxiety symptoms from multiple imputations. Indeed, difficulty in reaching and retaining individuals most in need of mental health support is not uncommon in such research. ${ }^{21}$

The high proportion of keyworkers in our cohort (50\% at baseline) may have also contributed to the high drop-out rate. These individuals were, by definition, providing essential services throughout the pandemic, and will therefore have had less capacity to remain engaged in the research. Another limitation includes the absence of health behaviour data (e.g. on physical activity, sleep quality, etc.), which may also be related to mental health. ${ }^{7,35}$

In conclusion, our findings indicate that the effects of the COVID-19 pandemic on mental health have been profound, and persisted throughout 2020. Despite modest improvements with the easing of restrictions, symptoms of anxiety and depression remained stubbornly higher than pre-pandemic levels. Consistent with previous work, being female, younger and having a previous history of mental health difficulties were associated with a greater risk of anxiety and depression symptoms. However, our findings on modifiable predictors (i.e. loneliness and positive mood) highlight potential avenues for intervention.

Ru Jia (D, MSc, Centre for Academic Primary Care, School of Medicine, University of Nottingham, UK; Kieran Ayling, PhD, Centre for Academic Primary Care, School of 
Medicine, University of Nottingham, UK; Trudie Chalder (D), PhD, Department of Psychological Medicine, Institute of Psychiatry, Psychology \& Neuroscience, King's college London, UK; Adam Massey, PhD, Centre for Academic Primary Care, School of Medicine, University of Nottingham, UK; Norina Gasteiger, MPH, Department of Psychological Medicine, University of Auckland, New Zealand; Elizabeth Broadbent PhD, Department of Psychological Medicine, University of Auckland, New Zealand; Carol Coupland, PhD, Centre for Academic Primary Care, School of Medicine, University of Nottingham, UK; Kavita Vedhara, PhD, Centre for Academic Primary Care, School of Medicine, University of Nottingham, UK

Correspondence: Kavita Vedhara. Email: kavita.vedhara@nottingham.ac.uk

First received 25 Jun 2021, final revision 4 Feb 2022, accepted 14 Feb 2022

\section{Supplementary material}

Supplementary material is available online at https://doi.org/10.1192/bjo.2022.34.

\section{Data availability}

Data will be deposited in the University of Nottingham data archive. Access to this data-set will be embargoed for a period of 12 months to permit planned analyses of the data-set. Afterward, it may be shared with the consent of the Chief Investigator. Extra data is available by contacting kavita.vedhara@nottingham.ac.uk.

\section{Acknowledgements}

We would like to thank all our participants and the NHS organisations who helped us to promote the study.

\section{Author contributions}

R.J., K.A., T.C., A.M., N.G., E.B., C.C. and K.V. contributed to the study design. R.J., K.A., A.M. and K.V. were responsible for coordination and management of recruitment. R.J., K.A., C.C. and K.V. contributed to the preparation and analysis of the data. R.J., K.A., T.C., A.M., N.G., E.B., C.C. and K.V. interpreted the data. R.J., K.A., T.C., A.M., N.G., E.B., C.C. and K.V. prepared and reviewed the final manuscript.

\section{Funding}

K.A. acknowledges financial support from the National Institute for Health Research (NIHR) School for Primary Care Research. T.C. acknowledges financial support from the Department of Health via the NIHR Specialist Biomedical Research Centre for Mental Health award to the South London and Maudsley NHS Foundation Trust and the Institute of Psychiatry at King's South London and Maudsley NHS Foundation Trust and the Institute of Psychiatry at King's
College London. C.C. acknowledges support from the NIHR Nottingham Biomedical Research Centre. The views expressed are those of the authors and not necessarily those of the NHS, the NIHR or the Department of Health and Social Care. No other funding supported the work described in this manuscript. The study sponsor did not play a role in the study design, collection, analysis and interpretation of data; the writing of the report; or the decision to submit the paper for publication.

\section{Declaration of interest}

None.

\section{References}

1 Xiong J, Lipsitz O, Nasri F, Lui LMW, Gill H, Phan L, et al. Impact of COVID-19 pandemic on mental health in the general population: a systematic review. J Affect Disord 2020; 277: 55-64

2 Lazarus RS, Folkman S. Stress, Appraisal, and Coping. Springer, 1984.

3 Prime Minister's Office. Prime Minister's Statement on Coronavirus (COVID-19): 10 May 2020. Prime Minister's Office, 2020 (https://www.gov.uk/government/ speeches/pm-address-to-the-nation-on-coronavirus-10-may-2020).

4 Office for Health Improvement and Disparities. COVID-19 Mental Health and Wellbeing Surveillance: Report. Office for Health Improvement and Disparities, 2020 (https://www.gov.uk/government/publications/covid-19mental-health-and-wellbeing-surveillance-report).

5 Fancourt D, Steptoe A, Wright L. The Cummings effect: politics, trust, and behaviours during the COVID-19 pandemic. Lancet 2020; 396(10249): 464-5.

6 O'Connor RC, Wetherall K, Cleare S, McClelland H, Melson AJ, Niedzwiedz CL, et al. Mental health and well-being during the COVID-19 pandemic: Iongitudinal analyses of adults in the UK COVID-19 mental health \& wellbeing study. Br J Psychiatry 2021; 218(6): 326-33.

7 Gasteiger N, Vedhara K, Massey A, Jia R, Ayling K, Chalder T, et al. Depression, anxiety and stress during the COVID-19 pandemic: results from a New Zealand cohort study on mental well-being. BMJ Open 2021; 11(5): e045325.
8 Jia R, Ayling K, Chalder T, Massey A, Broadbent E, Coupland C, et al. Mental health in the UK during the COVID-19 pandemic: cross-sectional analyses from a community cohort study. BMJ Open 2020; 10(9): e040620.

9 Palgi Y, Shrira A, Ring L, Bodner E, Avidor S, Bergman Y, et al. The loneliness pandemic: Ioneliness and other concomitants of depression, anxiety and their comorbidity during the COVID-19 outbreak. J Affect Disord 2020; 275: 109-11.

10 Li JB, Yang A, Dou K, Wang LX, Zhang MC, Lin XQ. Chinese public's knowledge, perceived severity, and perceived controllability of COVID-19 and their associations with emotional and behavioural reactions, social participation, and precautionary behaviour: a national survey. BMC Public Health 2020; 20: 1589.

11 Bonanno GA, Ho SM, Chan JC, Kwong RS, Cheung CK, Wong $\mathrm{CP}$, et al Psychological resilience and dysfunction among hospitalized survivors of the SARS epidemic in Hong Kong: a latent class approach. Health Psychol 2008; 27(5): 659-67.

12 Spitzer RL, Kroenke K, Williams JB, Löwe B. A brief measure for assessing generalized anxiety disorder: the GAD-7. Arch Intern Med 2006; 166(10): 1092-7.

13 Kroenke K, Spitzer RL, Williams JB, Löwe B. The Patient Health Questionnaire somatic, anxiety, and depressive symptom scales: a systematic review. Gen Hosp Psychiatry 2010; 32(4): 345-59.

14 Diener E, Wirtz D, Tov W, Kim-prieto C, Choi D-W, Oishi S, et al. New well-being measures: short scales to assess flourishing and positive and negative feelings. Soc Indic Res 2010; 97: 143-56.

15 Lumley $\mathrm{T}$, Diehr $\mathrm{P}$, Emerson S, Chen L. The importance of the normality assumption in large public health data sets. Annu Rev Public Health 2002; 23(1): 151-69.

16 National Collaborating Centre for Mental Health. The Improving Access to Psychological Therapies Manual. National Collaborating Centre for Mental Health, 2021 (https://www.england.nhs.uk/wp-content/uploads/2018/06/theiapt-manual-v5.pdf)

17 Park N. Estimates of the Population for the UK, England and Wales, Scotland and Northern Ireland. Office for National Statistics, 2021 (https://www.ons. gov.uk/peoplepopulationandcommunity/populationandmigration/populationestimates/datasets/populationestimatesforukenglandandwalesscotlandand northernireland).

18 Jeličić $\mathrm{H}$, Phelps $\mathrm{E}$, Lerner RM. Use of missing data methods in longitudinal studies: the persistence of bad practices in developmental psychology. Dev Psychol 2009; 45(4): 1195-9.

19 Kocalevent RD, Hinz A, Brähler E. Standardization of the depression screener Patient Health Questionnaire (PHQ-9) in the general population. Gen Hosp Psychiatry 2013; 35(5): 551-5.

20 Löwe B, Decker O, Müller S, Brähler E, Schellberg D, Herzog W, et al. Validation and standardization of the generalized anxiety disorder screener (GAD-7) in the general population. Med Care 2008; 46(3): 266-74.

21 Czeisler M, Wiley J, Czeisler C, Rajaratnam S, Howard M. Uncovering survivorship bias in longitudinal mental health surveys during the COVID-19 pandemic. Epidemiol Psychiatr Sci 2021; 30: e45.

22 Daly $M$, Sutin AR, Robinson E. Longitudinal changes in mental health and the COVID-19 pandemic: evidence from the UK household longitudinal study. Psychol Med [Epub ahead of print] 13 Nov 2020. Available from: https://doi.org/10.1017/S0033291720004432.

23 Davies J. What Impact has Covid-19 had on Mental Health Services? Nuffield Trust, 2020 (https://www.nuffieldtrust.org.uk/news-item/what-impact-hascovid-19-had-on-mental-health-services).

24 Bakolis I, Stewart R, Baldwin D, Beenstock J, Bibby P, Broadbent M, et al. Changes in daily mental health service use and mortality at the commencement and lifting of COVID-19 'lockdown' policy in 10 UK sites: a regression discontinuity in time design. BMJ Open 2021; 11(5): e049721.

25 Kaufman KR, Petkova E، Bhui KS, Schulze TG. A global needs assessment in times of a global crisis: world psychiatry response to the COVID-19 pandemic. BJPsych Open 2020; 6(3): e48.

26 Mughal F, Hossain MZ, Brady A, Samuel J, Chew-Graham CA. Mental health support through primary care during and after covid-19. BMJ 2021; 373: n1064

27 Rossi R, Jannini TB, Socci V, Pacitti F, Lorenzo GD. Stressful life events and resilience during the COVID-19 lockdown measures in Italy: association with menta health outcomes and age. Front Psychiatry 2021; 12: 635832.

28 Wright L, Steptoe A, Fancourt D. Does thinking make it so? Differential associations between adversity worries and experiences and mental health during the COVID-19 pandemic. J Epidemiol Community Health 2021; 75(9): 817-23.

29 Witteveen $D$, Velthorst E. Economic hardship and mental health complaints during COVID-19. Proc Natl Acad Sci U S A 2020; 117(44): 27277-84.

30 Goldman ML, Druss BG, Horvitz-Lennon M, Norquist GS, Kroeger Ptakowski K Brinkley A, et al. Mental health policy in the era of COVID-19. Psychiatr Serv 2020; 71(11): 1158-62. 
31 Pérez-Fuentes MDC, Molero Jurado MDM, Martos Martínez A, Gázquez Linares JJ. Threat of COVID-19 and emotional state during quarantine: positive and negative affect as mediators in a cross-sectional study of the Spanish population. PLOS One 2020; 15(6): e0235305.

32 Williams CYK, Townson AT, Kapur M, Ferreira AF, Nunn R, Galante J, et al. Interventions to reduce social isolation and loneliness during COVID-19 physical distancing measures: a rapid systematic review. PLOS One 2021; 16(2): e0247139.

33 Koydemir S, Sökmez AB, Schütz A. A meta-analysis of the effectiveness of randomized controlled positive psychological interventions on subjective and psychological well-being. Appl Res Qual Life 2021; 16(3): 1145-85.
34 Bendau A, Kunas SL, Wyka S, Petzold MB, Plag J, Asselmann E, et al Longitudinal changes of anxiety and depressive symptoms during the COVID19 pandemic in Germany: the role of pre-existing anxiety, depressive, and other mental disorders. J Anxiety Disord 2021; 79: 102377.

35 Yuksel D, McKee GB, Perrin PB, Alzueta E, Caffarra S, Ramos-Usuga D, et al. Sleeping when the world locks down: correlates of sleep health during the COVID-19 pandemic across 59 countries. Sleep Health 2021; 7(2): 134-42.

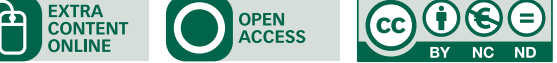

